\title{
Locus Minoris Resistentiae: Mycobacterium chelonae in Striae Distensae
}

\author{
Rachel M. Greenwood, MD; Morgan W. Thakore, MD; Loretta S. Davis, MD
}

\section{PRACTICE POINTS}

- Striae distensae, seen frequently in the setting of chronic corticosteroid use, are at an increased risk for localized infection, particularly in immunocompromised patients. There should be a low threshold to biopsy striae distensae that demonstrate morphologic evolution.

- The Koebner reaction, also known as an isomorphic response, refers to the appearance of certain dermatoses in previously healthy skin subjected to cutaneous injury.

- Locus minoris resistentiae is an isotropic response that characterizes the presentation of a new dermatosis within an area previously affected by an unrelated skin condition that has healed.

To the Editor:

Immunosuppressed patients are at particular risk for disseminated mycobacterial infections. A locus minoris resistentiae offers less resistance to the infectious spread of these microorganisms. We present a case of Mycobacterium chelonae infection preferentially involving striae distensae.

A 30-year-old man with chronic eosinophilic pneumonia requiring high-dose corticosteroid therapy presented with widespread skin lesions. He reported no history of cutaneous trauma or aquatic activities. Physical examination revealed the patient was markedly cushingoid with generalized cutaneous atrophy and widespread striae. Multiple erythematous papules surrounded a large ulceration on the dorsal aspect of the left hand. Depressed erythematous plaques and several small crusted erosions extended up the left lower leg (Figure 1) to the knee. Strikingly, numerous brown and pink papules and small plaques on the left thigh were primarily confined within striae (Figure 2).

A biopsy of the left thigh revealed granulomatous inflammation (Figure 3) with numerous acid-fast bacilli

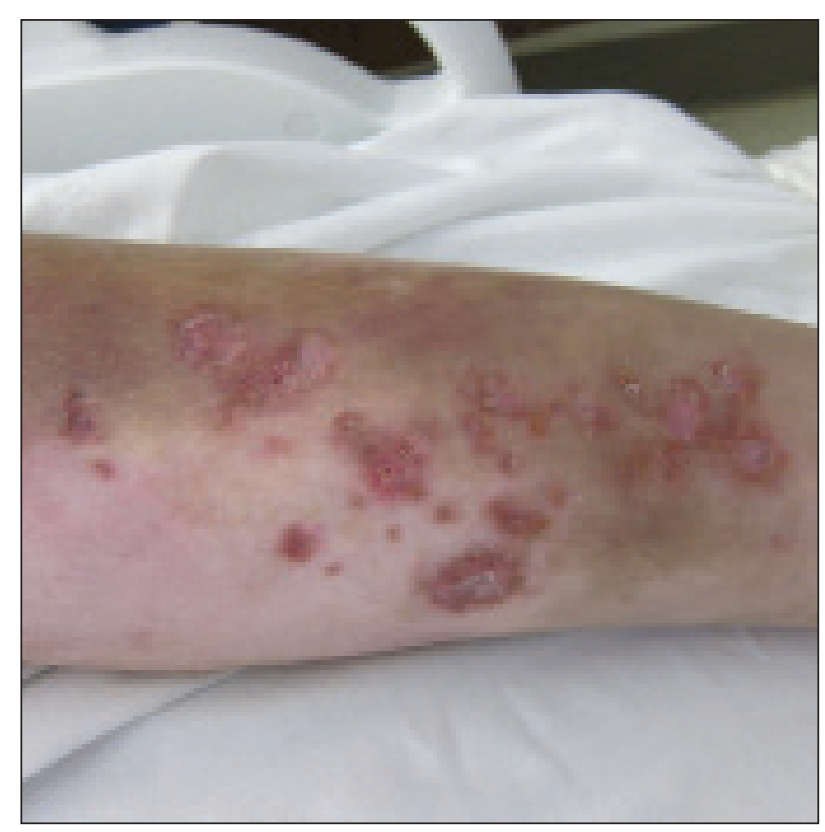

FIGURE 1. Erythematous depressed plaques with scale and a few crusted erosions on the anterior aspect of the lower leg. 


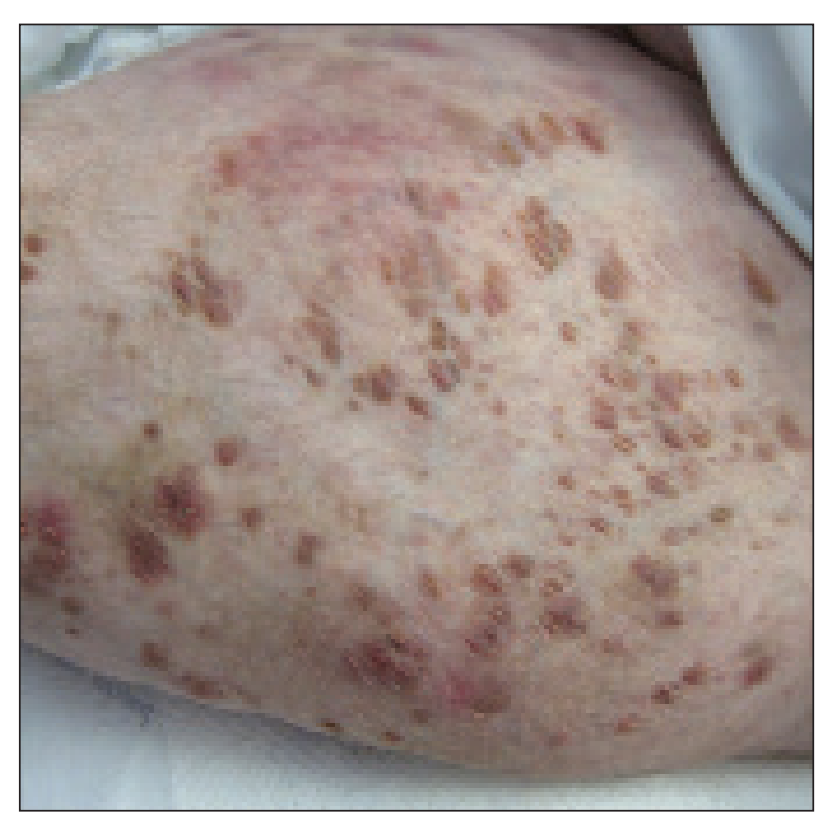

FIGURE 2. Markedly atrophic striae distensae on the left thigh were pockmarked with hyperpigmented and erythematous papules as well as small plaques.

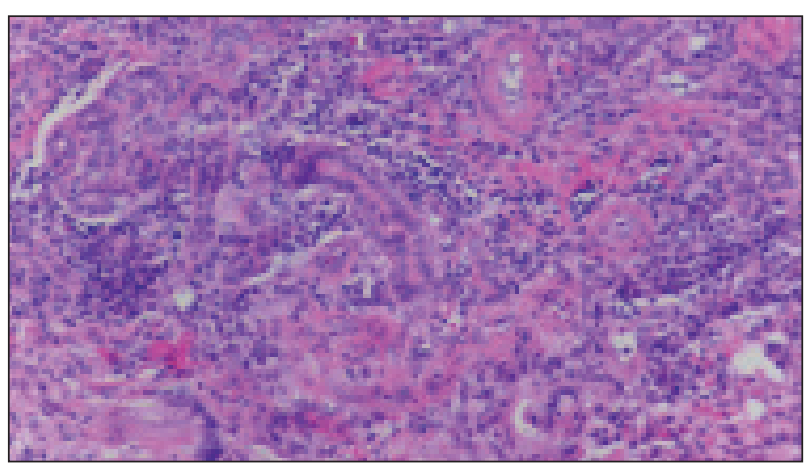

FIGURE 3. Histopathology demonstrated granulomatous inflammation within the subcutis (H\&E, original magnification $\times 40)$.

(Figure 4). Broad-spectrum coverage for fast-growing acid-fast bacilli with amikacin, ceftriaxone, levofloxacin, and clarithromycin was initiated with steady improvement of the eruption. Tissue culture subsequently grew Mycobacterium abscessus-chelonae, and therapy was narrowed to clarithromycin and moxifloxacin.

Mycobacterium chelonae is a rapidly growing mycobacteria isolated from soil and water worldwide, and human skin is a commensal organism. Cutaneous infections have been associated with traumatic injury, tattooing, surgery, cosmetic procedures, vascular access sites, and acupuncture. ${ }^{1}$ Most cases of cutaneous $M$ chelonae infection begin as a violaceous nodule. Over weeks to months, the localized infection progresses to multiple papules, nodules, draining abscesses, or ulcers. Infections tend to disseminate in immunosuppressed patients, and granulomatous inflammation may not be seen. ${ }^{1}$

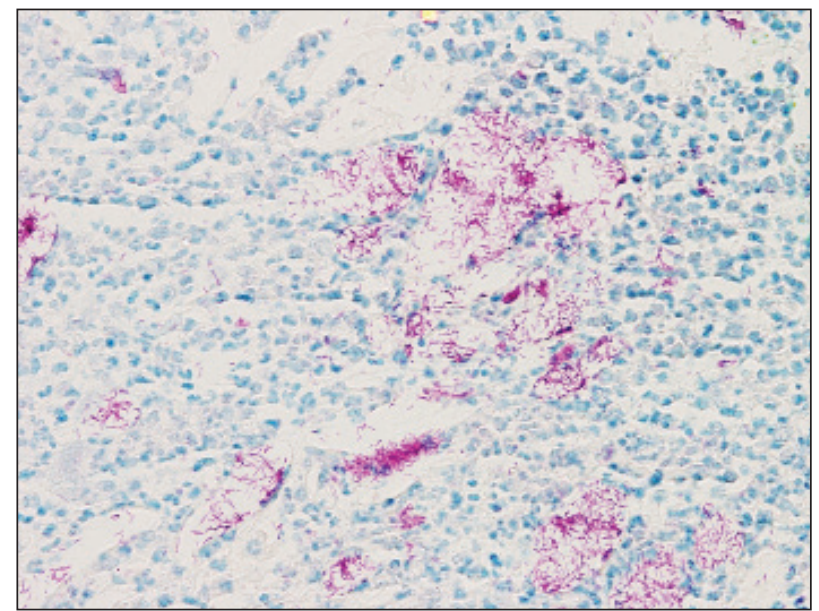

FIGURE 4. Acid-fast stain demonstrated numerous acid-fast bacilli (original magnification $\times 100$ ).

Atypical mycobacterial infection occurring within striae distensae is an example of locus minoris resistentiae-a place of less resistance. Wolf et $\mathrm{al}^{2}$ hypothesized that locus minoris resistentiae could explain the occurrence of an isotopic response or the occurrence of a new skin disorder at the site of a previously healed skin condition. They suggested that the same site could be affected by 2 unrelated diseases at different times due to an inherited or acquired susceptibility in the area. ${ }^{2}$ Herpes zoster serves as a primary example of Wolf phenomenon, as numerous conditions including granuloma annulare, pseudolymphoma, Bowen disease, and acne have reportedly emerged in its wake. ${ }^{3}$

Although locus minoris resistentiae does not specifically involve traumatized skin, it must be distinguished from the Koebner phenomenon, characterized by the appearance of isomorphic lesions in areas of otherwise healthy skin subjected to cutaneous injury, as well as the pseudo-Koebner phenomenon, a similar process involving infectious agents. ${ }^{3}$

In our patient, striae distensae represented areas of increased predisposition to infection. The catabolic effect of high corticosteroid levels on fibroblast activity decreased collagen deposition in the dermal matrix, leading to the formation of linear bands of atrophic skin. ${ }^{4}$ The elastic fiber network in striae distensae is reduced and reorganized compared to normal skin, in which an intertwining elastic system forms a continuum from the dermoepidermal junction to the deep dermis. ${ }^{5}$ The number of vertically oriented fibrillin microfibrils subjacent to the dermoepidermal junction and elastin fibers in the papillary dermis is comparatively diminished such that the elastin and fibrillin fibers in the deep dermis run more horizontally compared to normal skin. ${ }^{4}$ Consequently, collagen alignment in striae distensae demonstrates more anisotropy, or directionally dependent variability, and the dermal matrix is looser and more floccular than the surrounding skin. ${ }^{6}$ These alterations of dermal architecture 
likely provide a mechanical advantage for intradermal spread of $M$ chelonae within striae.

Other dermatoses have been observed to occur within striae distensae, specifically leukemia cutis, urticarial vasculitis, lupus erythematosus, keloids, linear focal elastosis, chronic graft-vs-host disease, psoriasis, gestational pemphigoid, and vitiligo. ${ }^{7}$ Given the dissimilarities of these conditions, the distinctive milieu of striae must provide an invitation-a locus minoris resistentiae-for secondary pathology.

Chronic corticosteroid use leads to both immunosuppression and striae distensae, effectively creating a perfect storm for an atypical mycobacterial skin infection demonstrating locus minoris resistentiae. The immunosuppressed state makes patients more susceptible to infection, and striae distensae may serve as a conduit for the offending organisms.

Acknowledgments - We are indebted to Letty Peterson, MD (Vidalia, Georgia), for her referral of this case, and to
Stephen Mullins, MD (Augusta, Georgia), for his dermatopathology services.

\section{REFERENCES}

1. Hay RJ. Mycobacterium chelonae - a growing problem in soft tissue infection. Cur Opin Infect Dis. 2009;22:99-101.

2. Wolf R, Brenner $\mathrm{S}$, Ruocco V, et al. Isotopic response. Int J Dermatol. 1995;34:341-348.

3. Medeiros do Santos Camargo C, Brotas AM, Ramos-e-Silva M, et al. Isomorphic phenomenon of Koebner: facts and controversies. Clin Dermatol. 2013;31:741-749.

4. Watson REB, Parry EJ, Humphries JD, et al. Fibrillin microfibrils are reduced in skin exhibiting striae distensae. $\mathrm{Br} J$ Dermatol. 1998;138:931-397.

5. Bertin C, A Lopes-DaCunha, Nkengne A, et al. Striae distensae are characterized by distinct microstructural features as measured by non-invasive methods in vivo. Skin Res Technol. 2014;20:81-86.

6. Elsaie ML, Baumann LS, Elsaaiee LT. Striae dístensae (stretch marks) and different modalities of therapy: an update. Dermatol Surg. 2009;35:563-573.

7. Liu $\mathrm{CI}, \mathrm{Hsu} \mathrm{CH}$. Leukemia cutis at the site of striae distensae: an isotopic response? Int J Dermatol. 1995;34:341-348. 